\title{
COMBATE BIOLÓGICO DE LA MUSTIA HILACHOSA (Thanatephorus cucumeris) EN EL FRIJOL EN PANAMÁ ${ }^{1}$
}

\author{
Emigdio Rodríguez-Quiel ${ }^{2}$, Ana Priscilla Montenegro-Alonso ${ }^{3}$, Jose Carlos Ureta-Reyes ${ }^{3}$, Norberto Pitty-Suira ${ }^{3}$, \\ Francisco Gonzáles-Guevara ${ }^{2}$,Jorge Muñoz-Fuentes ${ }^{2}$
}

\section{RESUMEN}

Combate biológico de la mustia hilachosa (Thanatephorus cucumeris) en el frijol en Panamá. El objetivo del presente trabajo fue determinar la capacidad biocontroladora de hongos y bacteria endofíticos en $T$. cucumeris (Frank) Donk en condiciones de campo. Se evaluó la aplicación de Trichoderma sp. (Cepa 2-Finca Margarita), Trichoderma sp. (Cepa 1-Colombia) y $M$. anisopliae (Colombia) en concentración de $2 \times 10^{7}$ conidias/ $\mathrm{ml}$ de agua); B. subtilis a razón de 0,335; 0,670; 1,005 y 1,340 g i.a./l agua + Clorotalonil 36 g i.a. /ha; Benomil 50 WP en dosis de $250 \mathrm{~g}$ i.a./ha y el testigo absoluto. Hubo significancia $(\mathrm{P} \leq 0,0003)$ entre los tratamientos, la de menor severidad de $T$. cucumeris se dio con la adición de $M$. anisopliae, seguido de B. subtilis con 1340 g i.a./l agua + Clorotalonil 36 g i.a/ha con $11,87 \%$ y Bacillus subtilis a 0,335 g i.a./l agua + Clorotalonil $36 \mathrm{~g}$ i.a/ha con 15,0\% . Los tratamientos donde hubo mayor severidad fueron $B$. subtilis 0,670 g i.a./1 agua + Clorotalonil 36 g i.a/ha, Trichoderma sp. (Cepa 2-Finca Margarita) y el testigo absoluto con 26,$2 ; 22,25 \%$ y $21,85 \%$ de severidad, respectivamente. La ecuación de regresión para el experimento $Y=2502,91$ $13,021 *$ Sev, fue significativa e indicó pérdidas de 13,02 kg por cada uno por ciento de incremento de la enfermedad. En el análisis marginal el tratamiento con mayor ganancia fue M. anisopliae, con un beneficio neto de $28,34 \%$.

Palabras clave: Trichoderma, Metarrhizium, Bacillus subtilis.

\begin{abstract}
The biological control of web blight (Thanatephorus cucumeris) in common bean in Panama. The objective of this work was to evaluate the capacity of Trichoderma sp., Metarrhizium anisopliae and endophytic bacteria of T. cucumeris (Frank) Donk under field conditions, for the biological control of web blight (T. cucumeris) in beans. Applications of Bacillus subtilis, Trichoderma sp. (Strain 2-Villa Margarita), Trichoderma sp. (Strain 1-Colombia) and M. anisopliae (Colombia) at concentration of $2 \times 107$ conidia/ $\mathrm{ml}$ of water), B. subtilis at rates of $0.335,0.670,1.005$ and $1.340 \mathrm{~g}$ ai / $\mathrm{ml}$ of water $+36 \mathrm{~g}$ ai Chlorothalonil /ha, benomyl $50 \mathrm{WP}$ with doses of $250 \mathrm{~g}$ ai / ha and a control treatment were conducted. Results revealed high differences ( $\mathrm{P} \leq 0.0003)$ between treatments; the treatment with lower severity was $M$. anisopliae, followed by $B$. subtilis with $1,340 \mathrm{~g}$ ai /ml of water + Chlorothalonil at $36 \mathrm{~g}$ ai/ha with $11.87 \%$ and B. subtilis to $0,335 \mathrm{~g}$ ai $/ 1$ of water + Chlorothalonil at $36 \mathrm{~g}$ ai/ha, with $15.0 \%$. The treatments affected to the greatest degree were $B$. subtilis with $0,670 \mathrm{~g}$ ai/l of water + Chlorothalonil with 36 $\mathrm{g}$ ai/ha, Trichoderma sp. (Strain 2-Villa Margarita) and the control treatment with $26.2 \%, 22.25 \%$ and $21.85 \%$ of severity, respectively. The regression equation for the experiment $\mathrm{Y}=$ 2502.91-13.021* Sev, was significant and indicated losses of $13.02 \mathrm{~kg}$ for each percent increase of the disease. The marginal analysis revealed that the treatment with highest gain was M. anisopliae, recording a net profit of $28.34 \%$.
\end{abstract}

Keywords: Trichoderma, Metarrhizium, Bacillus subtilis.

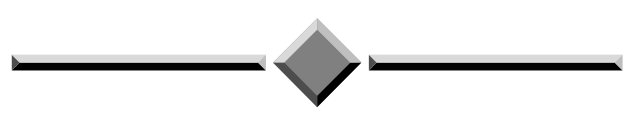

\footnotetext{
1 Recibido: 14 de mayo, 2011. Aceptado: 12 marzo, 2012. Trabajo que forma parte del proyecto Utilización de bioproductos y tecnologías limpias para la producción del frijol poroto en Panamá. Financiado por SENACYT.

2 Instituto de Investigación Agropecuaria de Panamá (IDIAP).erodriguezq15@yahoo.es; pancho125710@yahoo.com; j_alberto56@yahoo.es

3 Facultad de Ciencias Agropecuarias de Panamá. ana_priscilla@hotmail.com; juxc0413@hotmail.com; norbertopitty@gmail.com
} 


\section{INTRODUCCIÓN}

La mustia hilachosa cuyo agente causal es el hongo Thanatephorus cucumeris (Frank) Donk, es en el cultivo de frijol poroto (Phaseolus vulgaris L.), la limitante biótica de mayor importancia en el país (Lorenzo 2009). Se presenta en terrenos infectados con períodos prolongados de lluvias, temperatura promedio de $26^{\circ} \mathrm{C}$ y humedad relativa de $75 \%$ (Rodríguez et al. 1999).

Se considera a la mustia hilachosa como la enfermedad más destructiva del frijol, por la defoliación rápida y drástica que causa. Ataca follaje, tallos, ramas, granos y vainas de la planta de frijol en cualquiera de sus estados fenológicos. En condiciones favorables para el desarrollo de la enfermedad, puede causar una destrucción rápida de las plantas atacadas en una o dos semanas y pérdidas en el rendimiento de hasta 50\% (Rodríguez et al. 1997, Rodríguez et al. 1999).

El hongo sobrevive de una estación a otra por medio de esclerocios y micelio, en los residuos de cosecha, como saprófito de suelo o colonizando desechos de otros cultivos (Brenes 1996).

Para el manejo de este patógeno, Rodríguez y Lorenzo (2009) recomiendan el uso de fungicidas sintéticos, complementarios a la siembra de variedades de resistencia intermedia. Las prácticas agronómicas reducen el daño provocado por este patógeno, pero los fungicidas químicos afectan negativamente al medio ambiente y a la salud humana.

Una alternativa de gran potencial en el manejo de esta enfermedad, es el combate biológico, el cual se basa en la reducción de la densidad de inóculo o actividad infecciosa, por uno o más organismos antagónicos (Morales et al. 2007).

La cepa de Trichoderma spp. causó de 90 a $100 \%$ de mortalidad del hongo que causa la mustia hilachosa en frijol poroto, cuando se aplicó a un plato Petri por pote (Rijal y Shrestha 2002). El aislamiento proveniente de esclerocios refrigerados de $R$. solani resultó ser el más agresivo. El Trichoderma spp. proveniente del valle de Kathmandu resultó ser el antagonista de menor crecimiento. Al igual se demostró en experimentos de campo que aplicaciones al suelo de $100 \mathrm{~kg} /$ ha y tratamientos de semilla con 100 millones de conidias/mililitro/10 gramos de semilla lograron los más altos porcentajes de control de la mustia $(89,47 \%)$, lo que demuestra que a altas concentraciones del hongo, mejores pueden ser los resultados de control sobre la mustia hilachosa.

Plántulas de poroto inoculadas con B. subtilis, $B$. cereus o $B$. pumilus, provocaron una reducción en la incidencia de la enfermedad causada por Sclerotium rolfsii de $72 \%, 79 \%$ y $26 \%$ respectivamente; comparado con el control. Tanto en las plántulas de algodón como de poroto, estos endofíticos redujeron el índice de la enfermedad en más del $50 \%$. Estos resultados indican que las bacterias endofíticas pueden sobrevivir dentro de las plántulas y son agentes eficientes para el control biológico bajo condiciones de invernadero (Pleban et al. 1995). Esto indica que B. subtilis es eficiente en el control de hongos en el cultivo del frijol.

El objetivo de este trabajo fue evaluar la capacidad biocontroladora de hongos y bacteria endofíticos en T. cucumeris (Frank) Donk en condiciones de campo.

\section{MATERIALES Y MÉTODOS}

La prueba experimental en campo se estableció en la localidad de Caisán Centro, provincia de Chiriquí, Panamá a $08^{\circ} 46^{\prime} 03,7^{\prime \prime}$ de latitud norte y $82^{\circ} 47^{\prime}$ $29,9^{\prime \prime}$ de longitud oeste; durante los meses de octubre a enero, época tradicional para la siembra del poroto, el cual coincide con la época de mayor presión del inóculo de la enfermedad conocida como mustia hilachosa.

La semilla que se utilizó fue de la variedad criolla de poroto "Rosado", la cual posee un período vegetativo que varía entre 75 y 80 días, muestra bajos rendimientos y es susceptible a la mustia hilachosa.

El diseño experimental utilizado fue el de Bloques Completamente al Azar, nueve tratamientos con cuatro repeticiones. Las unidades experimentales constaban de $10 \mathrm{~m}^{2}(2,0 \times 5,0 \mathrm{~m})$, con cuatro surcos de $5 \mathrm{~m}$ de largo y separados a $0,50 \mathrm{~m}$.

Se evaluó la aplicación sobre las semillas del frijol poroto y luego la aspersión en el follaje de Trichoder$m a$ sp. (Cepa 2-Finca Margarita) en concentración de $2 \times 10^{7}$ conidias/ $\mathrm{ml}$ de agua; Trichoderma sp. (Cepa 1-Colombia) a dosis de 1500 g P.C/ha; Metarrhizium anisopliae (Colombia) a razón de 1000 g P.C/ha; $B a-$ cillus subtilis a razón de $0,335,0,670 ; 1,005$ y $1,340 \mathrm{~g}$ i.a./l agua + Clorotalonil 36 g i.a./ha; Benomil $50 \mathrm{WP}$ a dosis de $250 \mathrm{~g}$ i.a./ha y el testigo absoluto (sin aplicaciones). Las aplicaciones de Trichoderma sp. (Cepa 1- 
Colombia y Cepa 2-Finca Margarita), Trichoderma sp. y Metarrhizium anisopliae (Colombia) se realizaron a los 10, 25 y 40 días después de la emergencia (dde). En cambio los otros tratamientos fueron aplicados a los 15,30 y 45 dde.

La preparación de la cepa nativa de Trichoderma sp. (Cepa 2-Finca Margarita), Trichoderma sp. y $M$. anisopliae requirió de una suspensión de esporas. El hongo se incrementó en el medio nutritivo PDA (Papa Dextrosa Agar) durante 12-15 días a temperatura ambiente. Posteriormente se procedió a su extracción, adicionando agua destilada a los platos petri y raspando suavemente con un asa. Una vez desprendida la mayor cantidad de micelio y/o esporas, se procedió al conteo utilizando una cámara hematocitométrica de Neubauer, obteniendo suspensiones de estas cepas a la concentración deseada.

Durante el periodo de ejecución del ensayo se evaluaron las plantas emergidas, la severidad de la enfermedad a los 40 y 60 días después de la siembra (dds), plantas cosechadas y el rendimiento comercial a $14 \%$ de humedad.

Para la determinación de la severidad de la enfermedad se utilizó la escala de evaluación de Schoonhoven y Pastor (1987), la cual estima el porcentaje de infección de cada planta para luego calcular el promedio para toda la parcela.

Las variables cuantitativas evaluadas fueron analizadas con el programa SAS (2000). Se obtuvieron análisis de varianza (ANOVA), regresión y correlación. Para la diferenciación de medias, se utilizó el procedimiento de Duncan y de Tukey.

Con el objetivo de evaluar el progreso de la enfermedad a través del tiempo se obtuvo el área bajo la curva de progreso de la enfermedad (ABCPE), y se estimó mediante la fórmula:

$$
\mathrm{ABCPE}=\sum\left[\left(\chi_{\mathrm{I}}^{\mathrm{n}-1}+\chi_{\mathrm{i}=1}\right) / 2\right]\left(\mathrm{t}_{\mathrm{i}+1}-\mathrm{t}_{\mathrm{i}}\right)
$$

donde, $n$ es el número de evaluaciones, $\chi$ la proporción de la enfermedad y $\left(\mathrm{t}_{\mathrm{i}+1}-\mathrm{t}_{\mathrm{i}}\right)$ es el intervalo entre dos evaluaciones consecutivas (Campbell y Madden 1990).

La comparación del área bajo la curva de los diferentes tratamientos, se realizó con un análisis de varianza y la comparación de medias a través de la prueba de Duncan. Además se realizó un análisis marginal, el cual es un procedimiento para calcular las tasas marginales de retorno entre tecnologías, iniciando con la de más bajo costo a la siguiente de mayor inversión, y comparando las tasas de retorno contra una de mínima aceptación (Evans 2005).

\section{RESULTADOS Y DISCUSIÓN}

La primera variable evaluada en este ensayo fue la de plantas emergidas, la cual no presentó diferencias estadísticas. Esto indica que esta variable no tuvo influencia directa de los tratamientos y por lo tanto el ensayo inició con iguales condiciones para los nueve tratamientos evaluados.

La severidad fue evaluada mediante dos lecturas realizadas a los 40 y 60 días después de la siembra pero dicha información fue analizada estadísticamente en conjunto y no por separado; con la finalidad de determinar el efecto acumulativo que provoca esta enfermedad a lo largo del tiempo y así evaluar si hubo interacción entre ambos periodos de lectura.

Para realizar los análisis estadísticos de variables no paramétricas como la de severidad, se transformó la variable a raíz cuadrada $\sqrt{ }(\mathrm{Y}+1)$ con el propósito de normalizar los datos y homogenizar la varianza.

Los resultados para severidad evidencian que hubo significancia entre los tratamientos, los bloques, los periodos y en los tratamientos vs. los periodos de 40 y 60 días después de la siembra. En donde la probabilidad para tratamientos fue $\leq 0,0003$; para bloques $\leq 0,0001$; entre los periodos de $\leq 0,0001$ y para tratamientos vs. periodos de $\leq 0,0247$. El análisis mostró un coeficiente de variación de 12,29\% lo que indica la confiabilidad de la información colectada.

Hubo diferencia significativa $(\mathrm{P} \leq 0,0003)$ entre los tratamientos.

Entre periodos también se presentó alta significancia, lo que demuestra que existió interacción de la severidad a los 40 y 60 días después de la siembra y que los valores en dichos periodos fueron acumulativos y progresivos, por lo que el aumento o disminución observada al final del periodo de evaluación fue consecuencia exclusiva del efecto de los diferentes tratamientos evaluados. Además, indica que los valores obtenidos durante ambos periodos de lectura fueron diferentes debido a las condiciones climáticas que se presentaron, las cuales fueron favorables para el desarrollo de la enfermedad. 


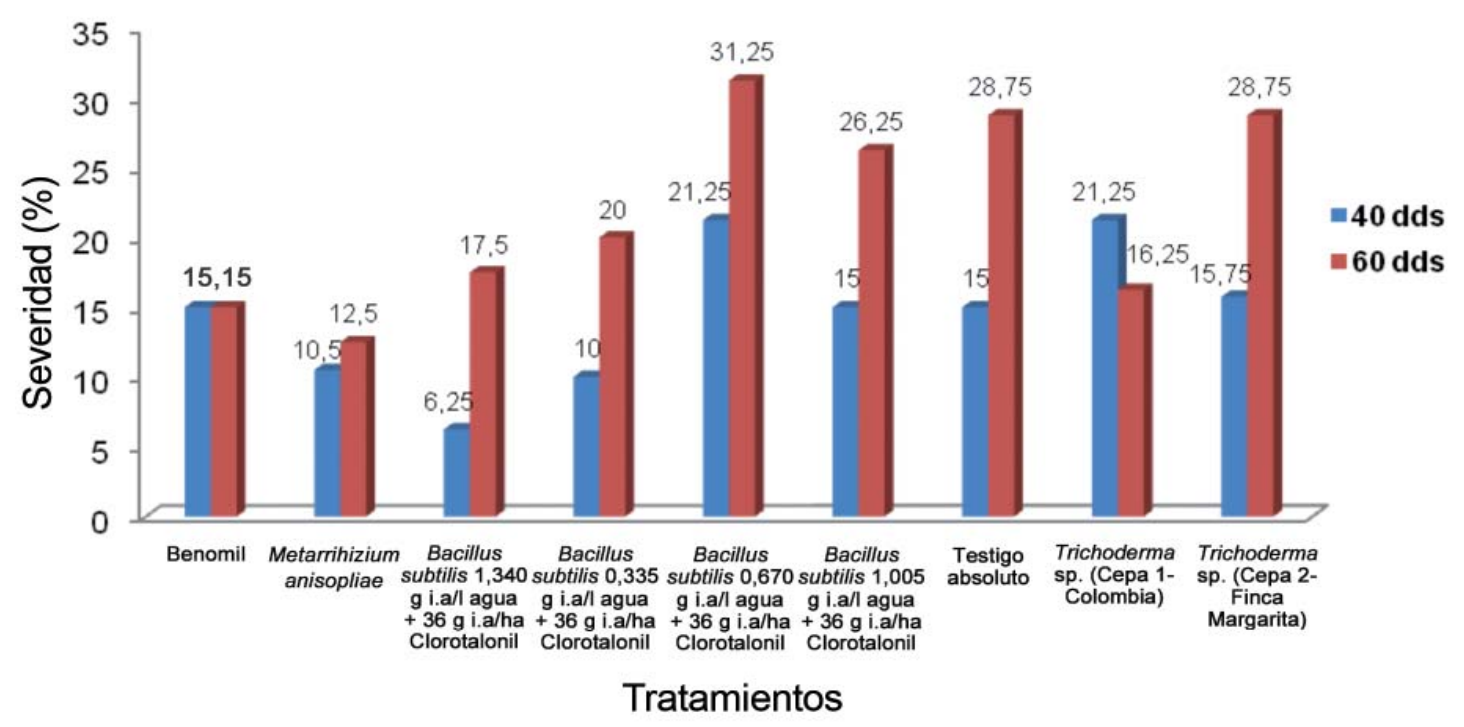

Figura 1. Severidad de T. cucumeris a los 40 y 60 días después de la siembra de frijol en la variedad criolla Rosado bajo tratamiento con hongos y bacterias endofíticas. Caisán, Panamá. 2009-2010.

La interacción tratamientos vs. periodos fue significativa, lo que demuestra que la enfermedad tuvo un efecto acumulativo y que los tratamientos aplicados a los 40 y 60 dds tuvieron un efecto sobre esta, ya sea reduciéndola, manteniéndola o incrementándola, lo que permitió establecer cuál de todos los tratamientos logró el mejor control de la enfermedad.

En la mayoría de los tratamientos se dio un aumento de la severidad de la enfermedad a lo largo del ciclo fisiológico del cultivo con excepción del tratamiento químico (benomil) (Figura 1); mientras que el tratamiento biológico a base de $M$. anisopliae logró los menores valores de área foliar afectada $(12,5 \%$ de severidad), el tratamiento a base de Trichoderma sp. (Cepa 1-Colombia) fue el que redujo el nivel de infección del patógeno en la planta y en la parcela. A pesar de que este tratamiento tuvo uno de los mayores porcentajes de severidad a los 40 dds fue de los mejores ya que redujo la infección, lo que sugiere que este tratamiento necesita aplicaciones más tempranas para un manejo de la enfermedad durante las fases iniciales de desarrollo de la misma.

$\mathrm{Al}$ igual que las plantas emergidas, los resultados del análisis de varianza para plantas cosechadas no fueron significativos ni entre tratamiento ni entre bloque. Esto indica que la severidad de la enfermedad no influyó en el número de plantas presentes al final del experimento. En cuanto a la variable de rendimiento los resultados del análisis de varianza indican que no existió diferencia significativa entre los tratamientos.

Los mejores rendimientos lo presentaron los tratamientos de $M$. anisopliae y Trichoderma sp. (Cepa 2-Finca Margarita) (Figura 2). En cambio, el tratamiento químico fue el que obtuvo el menor rendimiento en comparación con los demás; lo cual indica una tendencia a que los productos biológicos a base de bacterias y hongos endofíticos obtuvieron una mayor cantidad de kilogramos/hectárea de grano y a la vez se vieron afectados en menor grado por la enfermedad.

Para estimar el efecto de la severidad en el rendimiento del frijol poroto, se realizaron análisis de regresión lineal, por el origen y cuadrática, permitiendo de esta manera estimar valores cuando la ecuación mejor se ajustara. Este análisis se realizó de manera general, es decir, evaluando el efecto que provoca en el rendimiento final la severidad en ambos periodos.

El resultado del análisis de regresión lineal llevado a cabo de manera general indica que hubo significancia y que el modelo de regresión resultante fue $\mathrm{Y}=2502,91-13,021 *$ Sev. Esto nos indica que por cada grado de incremento de la severidad durante todo el desarrollo fisiológico del cultivo, se produjo 


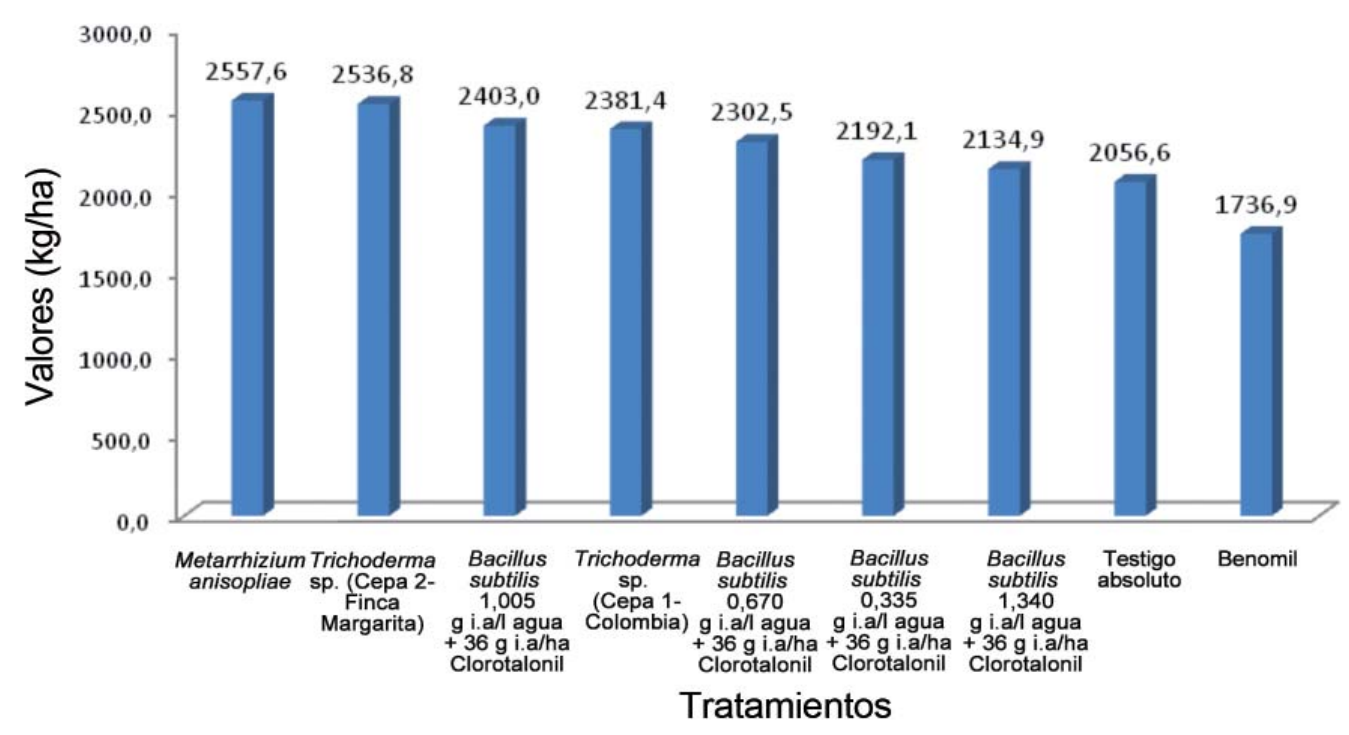

Figura 2. Rendimiento en $\mathrm{kg} / \mathrm{ha}$ al $14 \%$ de humedad en frijol variedad criolla Rosado, bajo protección contra T. cucumeris, con base en hongos y bacterias endofíticas. Caisán, Panamá. 2009-2010.

una reducción en el rendimiento de 13,02 kilogramos (Figura 3).

El análisis de regresión efectuado a los 40 dds muestra que con la adición de $B$. subtilis $1,005 \mathrm{~g}$ i.a/ ha+ Clorotalonil 36 g i.a./ha, M. anisopliae y Trichoderma sp. (Cepa 2-Finca Margarita) se obtuvo la menor reducción en el rendimiento, lo que significa que estos tratamientos controlaron en mayor grado al patógeno a los 40 días dds. En cambio, a los 60 dds los mayores rendimientos se obtuvieron con: $M$. anisopliae, $B$.

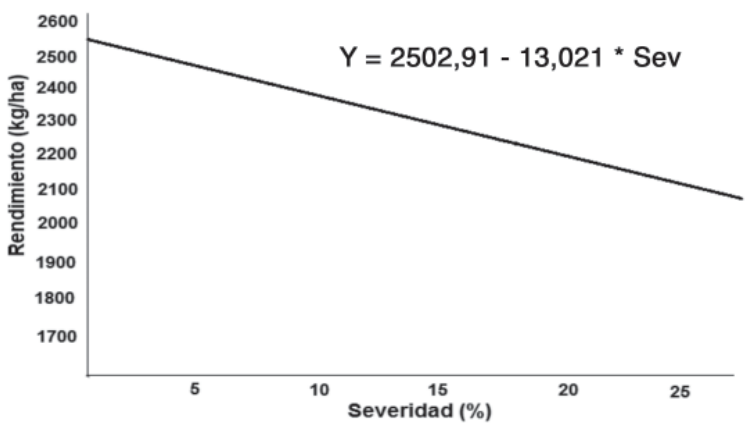

Figura 3. Modelo de regresión lineal resultante para el rendimiento vs. severidad de la mustia hilachosa a los 40 y 60 días después de la siembra, en la variedad Rosado de frijol criollo. Caisán, Panamá. 20092010. subtilis $1,005 \mathrm{~g}$ i.a/ha + clorotalonil $36 \mathrm{~g}$ i.a./ha y Trichoderma sp. (Cepa 1- Colombia); lo que demuestra que hubo una menor afección por parte del patógeno y por lo tanto pueden considerarse como los tratamientos más adecuados para el manejo de la enfermedad.

Al realizar el análisis de varianza del área bajo la curva de progreso de la enfermedad no se logró diferencia significativa entre los tratamientos.

La máxima severidad que se logró a los cuarenta días, en el tratamiento de Trichoderma sp. (Cepa 1-Colombia) y B. subtilis $0,335 \mathrm{~g}$ i.a/ha + clorotalonil $36 \mathrm{~g}$ i.a./ha, mientras que la menor fue en el de B. subtilis 1,005 g i.a./ha + clorotalonil 36 g i.a./ha. En cambio a los 60 dds la mayor afección se dio con el mismo tratamiento que en la primera lectura, el cual fue B. subtilis $0,335 \mathrm{~g}$ i.a/ha + clorotalonil $36 \mathrm{~g}$ i.a./ha, mientras que el menor porcentaje de daño se dio en el tratamiento de M. anisopliae (Figura 4).

En el periodo de veinte días que pasó entre la primera y segunda lectura hubo aumento en el porcentaje del área foliar afectada en todos los tratamiento con excepción del Trichoderma sp. (Cepa 1-Colombia), el cual redujo de $21,25 \%$ a $16,25 \%$ y el benomil se mantuvo en $15,0 \%$, lo que indica que solo el tratamiento a base de Trichoderma sp. es el que logra reducir el $\%$ de severidad del patógeno y así disminuir el daño producido a lo largo del ciclo fisiológico del cultivo. 


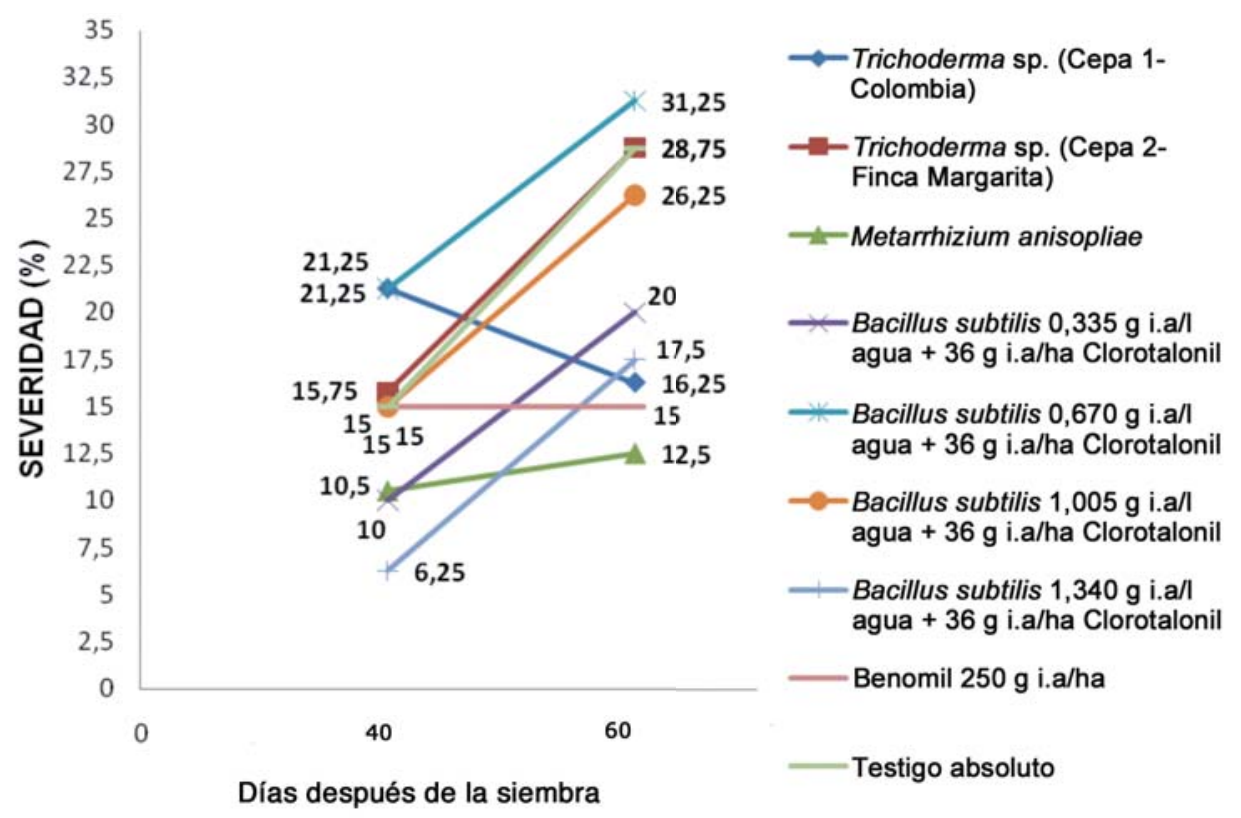

Figura 4. Área Bajo la Curva de Progreso de la Enfermedad causada por la mustia hilachosa bajo tratamientos de combate biológico con hongos y bacteria evaluados a los 40 y 60 días después de la siembra, en la variedad Rosado de frijol criollo. Caisán, Panamá. 2009-2010.

El tratamiento evaluado que resultó superior a los demás fue el de hongo endofítico M. anisopliae (Cepa 1 - Colombia), sin embargo es necesario considerar el costo y beneficio neto en comparación con otros tratamientos que no fueron superiores en cuanto al control de la enfermedad pero que resulten más rentables para los productores.

Debido a esto se efectuó un análisis marginal para obtener las tasas marginales de retorno (TMR), poder comparar los tratamientos y establecer cuál de ellos es el más conveniente para los productores de frijol poroto.

El Trichoderma sp. Cepa 1 y M. anisopliae constituyen cepas comerciales vendidas por una empresa comercial, mientras que Trichoderma sp. Cepa 2 es una cepa nativa colectada en Finca Margarita ubicada en Alanje y conservada en la estación del IDIAP (Instituto de Investigación Agropecuaria de Panamá) en Cerro Punta, Chiriquí (Cuadro 1).

En el análisis que se le realizó a todos los tratamientos evaluados, se excluyeron seis tratamientos (tratamientos dominados) porque implicaban mayores gastos y menores ganancias en comparación con otros tratamientos por lo cual ningún productor consideraría
Cuadro 1. Costo de los productos biológicos y químicos para el manejo de Thanatephorus cucumeris en frijol. Caisán, Panamá. 2009-2010.

\begin{tabular}{lcc}
\hline Tratamientos & Cantidad & $\begin{array}{c}\text { Precio/Unidad } \\
\text { (\$ U.S.) }\end{array}$ \\
\hline Bacillus subtilis & 11 & 11,70 \\
Clorotalonil & 11 & 10,90 \\
Benomil 50 WP & $1 \mathrm{~kg}$ & 16,00 \\
Trichoderma sp. (Cepa 1 & $1 \mathrm{~kg}\left(2,5 \times 10^{10}\right.$ & 21,00 \\
- Colombia) & esporas/gramo $)$ & \\
Trichoderma sp. (Cepa 2 & $2,0 \times 10^{7}$ coni- & 0,15 \\
- Finca Margarita) & dias/ml de agua & \\
Metarrhizium & $1 \mathrm{~kg}\left(2,5 \times 10^{10}\right.$ & 21,00 \\
anisopliae & esporas/gramo) & \\
\hline
\end{tabular}

utilizarlos. Los tres mejores fueron: testigo absoluto, Trichoderma sp. (Cepa 2 -Finca Margarita) y M. anisopliae.

$\mathrm{La}$ tasa de retorno marginal de comparar testigo absoluto con Trichoderma sp. (Cepa 2-Finca Margarita) es de $2295 \%$ y la de comparar Trichoderma sp. (Cepa 2-Finca Margarita) con M. anisopliae es de $10 \%$ (Figura 5). La mayor tasa de retorno marginal 


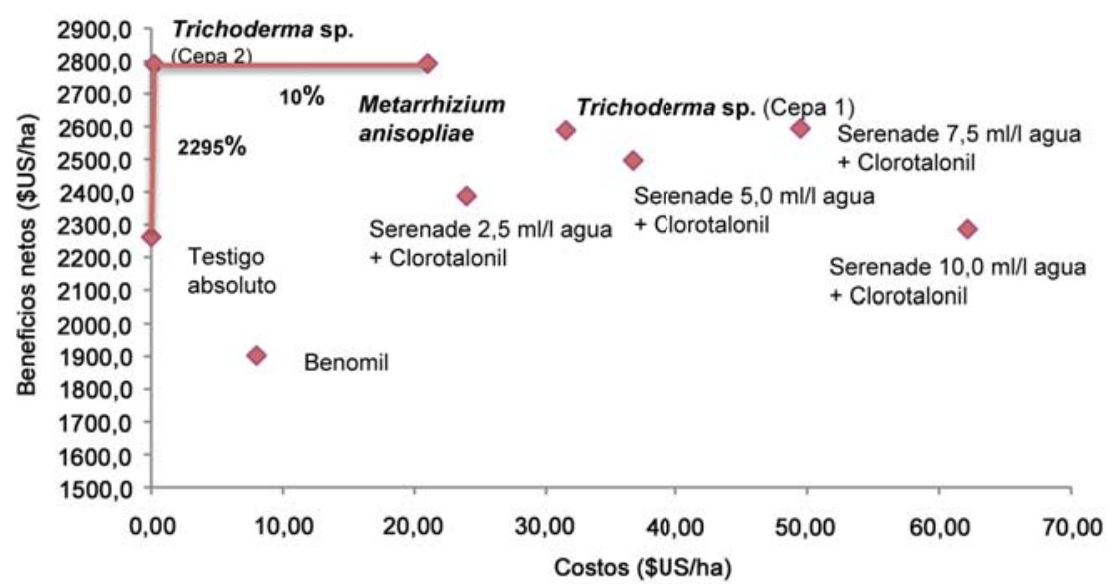

Figura 5. Curva de Beneficios Netos para todos los tratamientos de combate biológico de la mustia hilachosa en frijol criollo variedad Rosado. Caisán, Panamá. 2009-2010.

no indica que dicho tratamiento es el mejor, para esto es necesario comparar dichos valores con la tasa de retorno mínima aceptable para el agricultor, la cual se estableció que es de $50 \%$ ya que estos productos biológicos solo representan un ajuste de la práctica actual del agricultor y además es necesario calcular el residuo que resulta de sustraer el retorno requerido del beneficio neto.

El residuo para el testigo absoluto fue $\$ 2262$, para Trichoderma sp. (Cepa 2-Finca Margarita) fue de $\$ 2790$ y para M. anisopliae fue de \$2781, esto indica que el mejor tratamiento resultaría ser el de mayor residuo, sin embargo los dos tratamientos biológicos poseen poca diferencia entre los residuos y entre los beneficios netos por lo cual fueron prácticamente iguales en cuanto a rentabilidad.

El comportamiento general de los tratamientos para el manejo biológico de T. cucumeris indica que los productos utilizados ejercieron control del patógeno sin afectar la producción. La severidad de la enfermedad durante todo el ciclo fisiológico del cultivo tuvo un efecto significativo en la reducción del rendimiento final, en una relación 1:13.02. No hubo significancia para los parámetros evaluados de plantas emergidas, cosechadas y rendimiento comercial al $14 \%$ de humedad. El efecto de todos los tratamientos aplicados fue significativo en ambos periodos para la variable de severidad, lo que demuestra que la enfermedad tuvo un efecto acumulativo a lo largo del tiempo y que los tratamientos aplicados tuvieron efecto en el patógeno. El tratamiento más eficiente en la protección de la planta fue el del hongo endofítico M. anisopliae (Cepa 1-Colombia).

$\mathrm{El}$ alto porcentaje de severidad en la primera lectura después de la siembra en el tratamiento de Trichoderma sp. (Cepa 1-Colombia) y su posterior reducción en la segunda evaluación, indica que este hongo tuvo un efecto tardío en el control del patógeno.

\section{LITERATURA CITADA}

Brenes, BM. 1996. Epidemiología y manejo de la mustia hilachosa del frijol común causada por Rhizoctonia solani (en línea). San José, CR. Consultado 24 oct. 2009. Disponible en http://www.mag.go.cr/congreso_ agronomico_X/ a50-2388-II_031.pdf

Lorenzo, E. 2009. Manejo integrado de la mustia hilachosa en el cultivo de frijol poroto (Phaseolus vulgaris L.). s.e. IDIAP. 16 p.

Campbell, CL; Madden, LV. 1990. Introduction to plant disease epidemiology. s.e. New York, US. Wiley \& Sons. 532 p.

Evans, EA. 2005. Análisis marginal: un procedimiento económico para seleccionar tecnologías o prácticas alternativas (en línea). Florida, US. Consultado 9 abr. 2010. Disponible en http://edis.ifas.ufl.edu/fe573.

Morales, RA; Ríos, D; Muñoz, J; Concepción, R. 2007. Desarrollo y uso de bioproductos para el control de nematodos y sigatoka negra en plantaciones de plátano 
y banano: reporte final Proyecto IDIAP - FONTAGRO. s.e. 20 p.

Rodríguez, EQ; Lorenzo, E; De Gracia, R; González, GD; González, F. 1997. Manual técnico del manejo integrado del frijol común o poroto (Phaseolus vulgaris L.) en el sistema de mínima labranza. s.e. Panamá, PA. IDIAP. 75 p.

Rodríguez, E; Lorenzo, E; Acosta, M; González, F; Mora, B; Godoy, G. 1999. Manejo de la mustia hilachosa (Thanatephorus cucumeris (Frank)) en el cultivo del frijol común (Phaseolus vulgaris L.) (en línea). Agronomía Mesoamericana 10(1):99-108.

Rodríguez, EQ; Lorenzo, E. 2009. Manual técnico: tecnologías para el manejo integrado del cultivo de frijol poroto (Phaseolus vulgaris L.) en Panamá. s.e. Panamá, PA. IDIAP. 32 p.

Schoonhoven, A. Van; Pastor, M. 1987. Sistema estándar para evaluación de germoplasma de frijol (en línea). Cali, CO. Consultado 31 oct. 2009. Disponible en http://books.google.com/books?id=mpgIE_jDedMC $\& p g=$ PA 21\&lpg=PA21\&dq=CIAT+escalas+severid ad+frijol+1987\&source=bl\&ots=CJobIWDc0M\&sig =L_kigE4Lj3HhidRZX6WjpHdOpc\&hl=es\&ei=Dq7 nSsSLMMPe8Qbb7qWHBw\&sa=X\&oi=book_result $\& c t=$ result $\&$ resnum $=9 \& v e d=0 C D c Q 6 A E w C D g K \# v=$ onepage $\& \mathrm{q}=\& \mathrm{f}=$ false. 